\title{
Wilmot Voss K., 2014, The Food Section. Newspaper Women and the Culinary Community, Rowman \& Littlefield, Lanham, $252 \mathrm{p}$.
}

Kimberly Wilmot Voss, professeure de journalisme à la Nicholson School of Communication (université de Floride Centrale), s'intéresse dans The Food Section à un domaine généralement ignoré aussi bien par les historiens de la presse que par les historiens de la cuisine. Il s'agit des sections «alimentation » (food sections) des pages féminines des journaux américains ${ }^{1}$. Des années 1940 au milieu des années 1970, ces sections, qui traitent de nutrition, de recettes, de produits, de restaurants, représentent un volume important au sein des quotidiens américains (30 à 50 pages, notamment dans les éditions du week-end selon l'auteure) et elles emploient des équipes dédiées. À titre d'exemple, en 1955, Ruth Ellen Church, chef de la rubrique Food au Chicago Tribune, supervise sept personnes (cinq journalistes, un secrétaire et un assistant de cuisine). Après avoir vu leur volume doubler au sortir de la Seconde Guerre mondiale en raison du développement du marché de la publicité et de la multiplication des nouveaux produits alimentaires, les food sections disparaissent des journaux américains au cours des années 1970, remplacées par des sections style ou lifestyle.

L'étude des food sections des journaux américains permet non seulement de retracer une histoire de la cuisine domestique, mais elle permet aussi de mettre au jour le fonctionnement d'un monde journalistique dominé et méconnu. Le travail de K. Wilmot Voss se centre principalement sur cette seconde dimension. L'ouvrage comporte sept chapitres organisés selon une logique tantôt chronologique, tantôt thématique. Ils portent sur les origines du food journalism, sur son développement durant et après la Seconde Guerre mondiale (comme relais des messages politiques concernant l'alimentation), sur sa configuration dans un contexte de naissance des mouvements consuméristes, sur les livres de recettes écrits par ces journalistes, sur l'économie domestique, sur la critique de restaurant et enfin sur la disparition des food sections et les changements du métier. Ces chapitres présentent un intérêt inégal. Ce compte-rendu s'attachera aux éléments qui nous sont apparus comme les plus originaux.

\section{Qui étaient les journalistes culinaires ? Une approche par portraits}

Le livre se fonde avant tout sur des portraits de journalistes, ce qui présente un double avantage : d'une part, cela incarne ces rubriques et l'histoire racontée, et d'autre part, cela permet de sortir de l'oubli un certain nombre de femmes journalistes ayant connu une forte notoriété à leur époque (Jane

1 Les «pages féminines » regroupaient principalement les rubriques famille, mode, alimentation et décoration. Elles ont été inventées en 1891 par Joseph Pulitzer afin d'augmenter la publicité pour les produits féminins. 
Nickerson, Jeanne Voltz, Ruth Ellen Church, Cecily Brownstone, Poppy Cannon, Clementine Paddleford, etc.). La fin de l'ouvrage présente d'ailleurs utilement une courte biographie de soixante-cinq journalistes culinaires ayant exercé entre 1945 et 1975 .

Ces femmes journalistes sont ou bien des journalistes ayant reçu une éducation secondaire mais n'ayant pas trouvé de travail dans les informations générales, ou bien des diplômées d'économie domestique, ayant parfois suivi des cours de journalisme au sein de leur cursus. Créés en 1871, les programmes d'économie domestique avaient pour objectif d'apprendre aux jeunes filles à devenir de bonnes épouses et de bonnes mères. Progressivement, ils ont aussi ouvert à des débouchés professionnels (un doctorat a même été créé en 1930) dont le journalisme et la publicité. Dans la presse, les diplômées expérimentent les nouvelles technologies domestiques, écrivent des recettes et les testent dans les cuisines d'essai qui sont mises en place à partir des années 1920 et équipées par les industriels. Selon K. Wilmot Voss, ce sont les attaques féministes dénonçant le travail domestique et la cuisine comme une forme d'oppression de la femme qui signent l'arrêt de mort de l'économie domestique alors que paradoxalement, selon l'auteure, cette discipline, loin d'assigner les femmes aux tâches du foyer, a permis à celles-ci d'intégrer le monde professionnel et de faire carrière à une époque où le marché du travail leur était relativement fermé.

Du fait de leur formation, les journalistes culinaires mobilisent dans leur travail à la fois des compétences journalistiques (analyse de la législation, enquête de comparaison des prix, participation à des conférences, etc.) et des compétences techniques (réalisation de recettes). En analysant l'origine professionnelle des journalistes culinaires, l'auteure cherche aussi à remettre en question le "mythe " selon lequel le journalisme culinaire aurait été une activité de dilettantes jusqu'à l'arrivée de celui qui est considéré comme le premier «vrai » journaliste gastronomique, Craig Clairborne, le chef de la rubrique food du New York Times à partir de 1957. L'occultation contemporaine de l'héritage des food sections tenues par des journalistes femmes serait à l'origine de l'impression erronée de la nouveauté des rubriques food. Si cet héritage est occulté, c'est en partie parce que la spécialité avait une mauvaise réputation journalistique.

\section{Une spécialité journalistique dépréciée}

Les rubriques traitant de soft news (loisirs, culture, etc.) sont généralement dépréciées par rapport à celles des hard news (politique, économie, international, etc.). Outre que leur sujet est plus trivial, elles sont aussi, hier comme aujourd'hui, l'objet d'accusations concernant la compétence professionnelle de ceux qui y travaillent. À ce titre, l'ouvrage offre une très belle étude de cas. L'histoire de la profession des food journalists américains est en effet structurée autour d'une retentissante dénonciation publique de 
leur absence de moralité professionnelle. En 1971, Frank Moss, sénateur dirigeant le sous-comité des consommateurs du comité au commerce du Sénat américain, prend publiquement position à la conférence nationale des journalistes culinaires pour dénoncer leur absence de professionnalisme. Ils sont accusés de reprendre sans les modifier les communiqués de presse des industriels de l'agro-alimentaire, de ne pas défendre les consommateurs et de ne pas enquêter. Le sénateur recommande au gouvernement d'ouvrir une enquête officielle sur les pratiques des journalistes culinaires. Ces accusations publiques de corruption et de collusion, renforcées par la publication la même année d'un article de Robert Karp dans la Columbia Journalism Review dénonçant les conflits d'intérêt des journalistes culinaires, créent un tollé dans la profession et sont à l'origine de la structuration du groupe professionnel. Les journalistes décident de s'organiser pour se défendre en créant en 1974 leur première association professionnelle (Association of Food Journalists).

\section{La place des femmes dans le monde journalistique}

À partir d'un point d'entrée relativement étroit (les sections alimentation des women's pages des quotidiens américains), $\mathrm{K}$. Wilmot Voss retrace également une histoire de la place des femmes dans le journalisme américain au $\mathrm{XX}^{\mathrm{e}}$ siècle. Ainsi, elle souligne que jusqu'aux années 1960, les women's pages sont l'un des rares espaces du monde journalistique où les femmes journalistes peuvent avoir du pouvoir. Leur position subordonnée est toutefois marquée par le fait qu'elles sont parfois physiquement exclues des salles de rédaction. Ainsi, Associated Press fait-elle construire une cuisine-test au domicile de sa food writer Cecily Brownstone afin que cette dernière n'ait pas à travailler dans les locaux de l'agence.

Dans le monde journalistique, ce n'est qu'à partir de 1969 que les femmes commencent à être acceptées dans les organisations professionnelles comme le National Press Club. La progressive reconnaissance du caractère professionnel de leur activité va de pair avec le développement de l'idée selon laquelle les femmes doivent être employées dans toutes les sections des journaux et non cantonnées aux seules pages «féminines ». L'application de cette idée a toutefois un effet paradoxal. Elle conduit de fait à l'éviction des femmes des quelques positions dominantes qu'elles pouvaient détenir jusque-là à la tête des rubriques food, puisque ces dernières sont remplacées par des rubriques plus neutres du point de vue du genre (style) souvent dirigées par des hommes.

\section{Comment enquêter sur les food sections?}

Si les apports de l'ouvrage concernant l'histoire des médias, des femmes et de l'alimentation sont indéniables, la rigueur de la méthode d'enquête peine parfois à convaincre. Bien que l'auteure s'attache à décrire précisément les différentes sources mobilisées (archives, entretiens, études universitaires 
existantes, livres de cuisine édités par les journaux ou les journalistes étudiées, etc.), la manière dont elle a construit et délimité son échantillon reste floue. Surtout, l'ouvrage procède comme un recueil d'anecdotes, par une accumulation d'illustrations à partir de cas particuliers sans jamais qu'il soit indiqué si les cas évoqués sont représentatifs et dans quelle mesure il est possible de monter en généralité. Par exemple, K. Wilmot Voss postule que les journalistes réalisent des enquêtes avant d'écrire leurs articles. Les quelques exemples donnés tendent plutôt à renforcer l'idée que, la plupart du temps, ce n'est pas le cas. À l'issue de la lecture, le lecteur a ainsi le sentiment d'être noyé sous des cas particuliers (beaucoup de journaux évoqués, beaucoup de journalistes cités) sans avoir de vision globale étayant les assertions. Ce sentiment est renforcé par l'absence de définition claire des notions et des concepts employés (comment définit-on un food journalist par exemple ?) et par les va-et-vient chronologiques.

L'autre réserve quant à la méthodologie de l'auteure tient à ses partis pris. L'objectif de K. Wilmot Voss dans cet ouvrage est de réhabiliter des journalistes qu'elle considère avoir été injustement oubliées par l'histoire parce que femmes, parce que spécialistes d'un domaine trivial et parce que discréditées comme emblématiques d'une vision de la femme contraire à celle promue par les féministes dans les années 1970. S'il est aisé de suivre l'auteure dans sa volonté de mettre en lumière un pan occulté du monde social et le sérieux du travail des food journalists, en revanche, ce travail de réhabilitation s'assortit souvent d'un manque de distance par rapport à l'objet. Ainsi, les critiques adressées au travail des journalistes culinaires sont-elles systématiquement présentées comme fausses et non avenues, quand bien même rien dans le matériau présenté ne vient étayer ce point de vue de l'auteure. L'affirmation par l'auteure que l'indépendance à l'égard des annonceurs est plus importante dans les journaux (qui sont l'objet de l'étude) que dans les magazines demande ainsi à être fermement étayée, tant elle ne va pas de soi. De même, le seul argument pour contrer l'accusation d'absence d'indépendance des journalistes à l'égard de l'industrie (le fait que les journalistes sont assez intelligents pour voir au-delà du message publicitaire) peine à convaincre quand il est précisé par ailleurs que dans certains journaux, comme au Los Angeles Times jusqu'en 1960, c'est le département publicité qui publie la section food. Un tel postulat aurait mérité la mise en place d'un dispositif de vérification plus substantiel pour être validé (interviews avec des représentants de l'industrie et des attachés de presse, mesure de la couverture médiatique des produits dont on sait qu'ils ont été l'objet d'une promotion, etc.).

Un regard de sociologue peut aussi pousser le lecteur à regretter qu'une étude plus fine du travail concret des journalistes, des relations entre les acteurs et des contenus publiés n'ait été menée.

Finalement, l'ouvrage de $\mathrm{K}$. Wilmot Voss, en dépit des réserves qui peuvent lui être adressées, présente l'indéniable avantage de non seulement remettre en question des préjugés quant au rôle des femmes dans la sphère 
professionnelle, mais aussi de relativiser la «nouveauté » des rubriques culinaires actuelles et des questionnements qui les entourent. Il est à ce titre intéressant de noter la forte parenté entre les food journalists de l'après-guerre et les blogueurs culinaires contemporains. Dans les deux cas, les principaux sujets abordés dans les publications sont la cuisine domestique et ce qui l'entoure (famille, vie locale, etc.) et surtout, la relation établie avec le lectorat est une relation d'apparente proximité fondée sur des échanges intenses et un rôle d'animateur de communauté (de la même manière que les posts de blogs reçoivent des dizaines de commentaires, certaines food sections recevaient une centaine de lettres de lectrices par semaine). Si les dispositifs changent, les manières de publiciser la cuisine domestique semblent en revanche pérennes.

\author{
Sidonie NAULIN \\ PACTE, Sciences Po Grenoble \\ sidonie.naulin@iepg.fr
}


